\title{
(อ) OPEN ACCESS \\ Video-based reflection on neonatal interventions during COVID-19 using eye-tracking glasses: an observational study
}

\author{
Michael Wagner (1) , ${ }^{1}$ Maria C den Boer 다, ${ }^{2}$ Sophie Jansen, ${ }^{2}$ Peter Groepel, ${ }^{3}$ \\ Remco Visser, ${ }^{2}$ Ruben S G M Witlox, ${ }^{2}$ Vincent Bekker, ${ }^{2}$ Enrico Lopriore (ㅇ, ${ }^{2}$ \\ Angelika Berger, ${ }^{1}$ Arjan B te Pas $^{2}$
}

\begin{abstract}
Additional supplemental material is published online only. To view, please visit the journal online (http://dx.doi. org/10.1136/archdischild2021-321806)
\end{abstract}

${ }^{1}$ Department of Pediatrics, Comprehensive Center for Pediatrics, Medical University of Vienna, Vienna, Austria

${ }^{2}$ Department of Pediatrics, Leiden University Medical

Center, Leiden, The Netherlands ${ }^{3}$ Department of Applied

Psychology, University of Vienna, Vienna, Austria

Correspondence to DDr Michael Wagner, Department of Pediatrics, Comprehensive Center for Pediatrics, Medical University of Vienna, Vienna, Austria; michael.b.wagner@meduniwien. ac.at

Received 6 February 2021 Accepted 16 June 2021 Published Online First 19 August 2021

\section{ABSTRACT}

Objective The aim of this study was to determine the experience with, and the feasibility of, point-ofview video recordings using eye-tracking glasses for training and reviewing neonatal interventions during the COVID-19 pandemic.

Design Observational prospective single-centre study. Setting Neonatal intensive care unit at the Leiden University Medical Center.

Participants All local neonatal healthcare providers. Intervention There were two groups of participants: proceduralists, who wore eye-tracking glasses during procedures, and observers who later watched the procedures as part of a video-based reflection.

Main outcome measures The primary outcome was the feasibility of, and the proceduralists and observers' experience with, the point-of-view eye-tracking videos as an additional tool for bedside teaching and video-based reflection.

Results We conducted 12 point-of-view recordings on 10 different patients (median gestational age of $30.9 \pm 3.5$ weeks and weight of $1764 \mathrm{~g}$ ) undergoing neonatal intubation $(n=5)$, minimally invasive surfactant therapy $(n=5)$ and umbilical line insertion $(n=2)$. We conducted nine video-based observations with a total of 88 observers. The use of point-of-view recordings was perceived as feasible. Observers further reported the point-of-view recordings to be an educational benefit for them and a potentially instructional tool during COVID-19.

Conclusion We proved the practicability of eyetracking glasses for point-of-view recordings of neonatal procedures and videos for observation, educational sessions and logistics considerations, especially with the COVID-19 pandemic distancing measures reducing bedside teaching opportunities.

\section{Check for updates}

(C) Author(s) (or their employer(s)) 2022. Re-use permitted under CC BY-NC. No commercial re-use. See rights and permissions. Published by BMJ.

To cite: Wagner M, den Boer $\mathrm{MC}$, Jansen $\mathrm{S}$, et al. Arch Dis Child Fetal Neonatal Ed 2022;107:F156-F160.

\section{BACKGROUND}

Many countries introduced social distancing interventions to prevent the spread of the novel SARSCoV-2. ${ }^{12}$ This response inevitably led to a decline in bedside teaching ${ }^{3}$ and training opportunities ${ }^{4}$ for students and residents. Innovative technologies might facilitate the training of healthcare providers and students without direct bedside attendance. ${ }^{4}$ There are currently numerous available options for maintaining procedural proficiency, such as webinars, virtual simulation and

\section{What is already known on this topic?}

- Recording and reviewing neonatal stabilisation during video-based observations provides numerous educational benefits.

- The COVID-19 pandemic led to a decline in opportunities for bedside teaching as well as training opportunities for students and residents.

- Eye-tracking methodology allows researchers to objectively measure gaze patterns of healthcare providers from a first-person view.

\section{What this study adds?}

The eye-tracking methodology was introduced during the COVID-19 pandemic to enable healthcare staff to witness procedures remotely while still having a first-person view.

- It is feasible to use eye-tracking glasses for point-of-view recordings and video reflections of neonatal procedures.

- Eye tracking has the potential to involve healthcare staff in more procedures and close significant gaps in learning neonatal procedures.

videoconferences. ${ }^{5}$ Besides supporting procedural proficiency, recording and reviewing neonatal stabilisation provides numerous educational advantages. ${ }^{78}$ However, allowing medical students and trainees to benefit from this opportunity during the COVID-19 pandemic can be challenging. Furthermore, providers attending video-based observations on neonatal stabilisation anticipated that reviewing other procedures at the neonatal intensive care unit (NICU), most importantly, neonatal intubations, would also be beneficial for improving the quality of neonatal care. ${ }^{7}$ The use of modern eye-tracking glasses with the option of real-time recording and streaming has recently emerged as a revolutionary method in procedural education. ${ }^{9-11}$ The eye-tracking methodology allows researchers to objectively measure healthcare providers' gaze patterns from a first-person view, thereby understanding the clinical reasoning process during task performance. $^{12-14}$ Furthermore, the eye-tracking 
methodology may also aid in the proper documentation of clinical information, as accurate and complete documentation of physiological parameters during neonatal stabilisations continues to be a challenge. ${ }^{15}$ The practice of eye tracking for reviewing purposes has not yet been studied and may improve medical education during the pandemic. This research project aimed to determine the experience with, and the feasibility of, pointof-view video recordings using eye-tracking glasses for training and reviewing neonatal interventions in the NICU. This project focused on video summarisation of point-of-care neonatal interventions during COVID-19, especially while complying with social distancing measures, and analysing, discussing and improving individual approaches and strategies for different neonatal procedures.

\section{METHODS}

This observational prospective single-centre study was conducted at the Leiden University Medical Center in a single-room NICU ward from September to November 2020 and was designed and described following the Strengthening the Reporting of Observational Studies in Epidemiology guidelines for observational studies. ${ }^{16}$ All neonatal healthcare providers (physician assistants, residents, fellows, attendees) were eligible to participate in the study. There were two groups of participants: (A) proceduralists, who wore eye-tracking glasses during procedures, and (B) observers who later watched the procedures as part of a video-based reflection. The procedures included endotracheal intubation, minimally invasive surfactant therapy (MIST), and umbilical catheter insertion. Proceduralists and healthcare professionals present in the room gave their consent for the recording, and parental consent was obtained to use the footage during observations. After providing consent, proceduralists were equipped with mobile eye-tracking glasses (VPS 19, Viewpointsystem, Vienna, Austria). The recording started after glasses calibration according to the manufacturer's recommendations. On the conclusion of the neonatal procedure, each proceduralist filled in a questionnaire to assess eye-tracking glasses' usability throughout said procedure. The neonatal procedures were carried out using the local medical equipment and following the standard operating practices.

\section{Video-based reflection on neonatal procedures}

The principal investigator of this study prepared and chaired the video-based observations. All healthcare staff of the NICU were invited to join as observers. During video reflections, recordings of the eye-tracker in a point-of-view first-person perspective were reviewed to evaluate care quality. Observers watched the full point-of-view video without audio and with the possibility to pause to discuss any potential issue. If available, videolaryngoscopy (InfantView, Vyaire, Chicago, Illinois, USA) recordings were integrated with the eye-tracking recording using the same format as during video reflections on neonatal stabilisation, performed weekly at the local NICU. ${ }^{8}$ Healthcare staff openly addressed both what went right and what could be improved. To conclude the video reflection, healthcare staff discussed what they had learnt. Videos were also streamed online via Microsoft Teams (Microsoft, Redmond, Washington, USA) for healthcare staff working from home.

\section{Eye-tracking glasses}

VPS 19 European CE-certified eye-tracking glasses (Viewpointsystem) were used to measure the proceduralists' visual field. The glasses include binocular eye-tracking cameras that record a real-time point-of-view first-person perspective and participants' gaze behaviour at a sampling rate of 30 frames per second. The eye tracker has a $70^{\circ}$ field of view and includes a $9 \mathrm{df}$ sensor with a three-axis accelerometer, magnetometer and gyroscope. The front camera records in full high definition (1080p). This study did not require audio recording. The glasses weigh $43 \mathrm{~g}$. The eye-tracking glasses were connected with a USB-C cable to a smart unit with a Linux-based operating system, which can be used to record and stream videos. All recordings were pseudonymised and labelled with a unique study ID.

\section{Primary outcome}

The primary outcome was the feasibility of, and the proceduralists and observers' experience with, the point-of-view eyetracking videos as an additional tool for bedside teaching and video-based observation. Proceduralists and observers filled in a questionnaire to evaluate their experience, the feasibility and potential limitations of wearing eye-tracking glasses.

\section{Secondary outcomes}

The secondary outcome was gauging how time consuming the set-up and calibration phase of the device was. In addition, we asked observers to report what they had learnt during the procedures.

\section{Questionnaire}

Both proceduralists and observers were asked to give feedback on the point-of-care video recording using a short questionnaire. For proceduralists, the questionnaire consisted of 14 questions about the feasibility of the eye-tracking glasses, perceived distractibility, discomfort while wearing the glasses and experienced impact on performance. Observers answered seven questions about their experience with the eye-tracking recordings (see online supplemental appendix), rating all items on a scale from 1 ('strongly disagree') to 5 ('strongly agree'). A supplementary open-ended question was used to describe what they had learnt from the video-based observation. On-site participants completed a paper-pencil questionnaire, while participants joining the video-based observation online submitted their questionnaire through a SurveyMonkey link (SurveyMonkey, San Mateo, California, USA).

\section{Statistical analysis}

Descriptive analysis was used to describe the study sample. A one-sample Wilcoxon signed-rank test was performed to test whether proceduralists' subjective experience with eye-tracking glasses was different from 3 (=the scale median), whereas onesample t-tests were used to analyse observers' ratings. For the one-sample t-test, which is the main analysis, an a priori power analysis predicted that a total sample size of 34 participants would give sufficient power $(0.80)$ to detect significant effects at the alpha level of 0.05 by medium effect size (Cohen's $d=0.50$ ). All quantitative analyses were performed with SPSS V.24.0 (IBM). The level of significance was set at $\mathrm{p}<0.05$ (two tailed). Observers' responses to what they had learnt from the recordings were analysed qualitatively using content analysis.

All parents of recorded children signed a written informed consent for the use of the recordings for educational and scientific purposes.

\section{RESULTS}

We conducted 12 point-of-view recordings (figure 1) on 10 different patients undergoing neonatal intubation $(n=5)$, MIST 


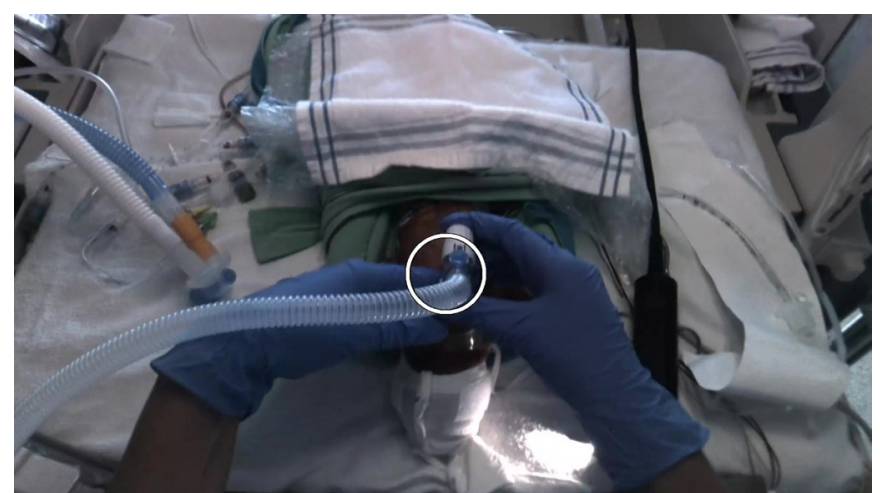

Figure 1 Snapshot of an eye-tracking video showing a healthcare provider performing positive pressure ventilation. The white circle indicates the visual focus of the provider.

$(n=5)$ and umbilical line insertion $(n=5)$. With these recordings, we conducted nine video-based observations (three on-site only and six on-site and online simultaneously) with a total of 88 observers filling in the questionnaire after the video-based observation. We included a short video clip with parts of recordings of a neonatal intubation, an umbilical line insertion and a MIST procedure as online supplemental material.

\section{Patients}

The recordings were performed on 10 patients with a median gestational age of $30.9 \pm 3.5$ weeks at the time of the procedure and a weight of $1764 \mathrm{~g}$. The diagnoses were asphyxia, prematurity, respiratory distress syndrome, double outlet right ventricle or transposition of the great arteries. Indications for procedures were presumed sepsis, necrotising enterocolitis, increased oxygen demand, atelectasis, respiratory insufficiency due to prematurity and preparation for surgery. Videolaryngoscopy was required in 10 intubation/MIST procedures.

\section{Proceduralists' responses}

All 12 proceduralists agreed to participate and completed the questionnaire. None of them removed the eye-tracking glasses during the procedures. The use of the glasses was perceived as feasible and non-hindering, as indicated by proceduralists' responses that were largely different from the scale median (figure 2). Proceduralists also reported no discomfort related to the glasses or performance alteration, neither positively nor negatively. The set-up and calibration duration of the eye-tracking glasses ranged from 1 to $10 \mathrm{~min}$, with a mean time of $3.5 \mathrm{~min}$.

Additionally, qualitative feedback from proceduralists included statements such as 'the glasses did not bother at all', '... were a good experience', but also that if used for an extended period (eg, during umbilical line insertion), 'the battery and smart unit were getting hot and heavy to wear'.

\section{Observers' responses}

We conducted nine video-based observations with a total of 88 observers. Three observations (29 observers) were held on-site and another six in a combined on-site (51 observers) and streaming (8 observers) format. The video reflections lasted from 20 to $30 \mathrm{~min}$, with a mean duration of $22.1 \mathrm{~min}$. The use of point-of-view recordings was perceived feasible. The median score of most questionnaire items was significantly different from the scale median (figure 3). Observers deemed the point-of-view recordings an educational benefit for them and the recordings an
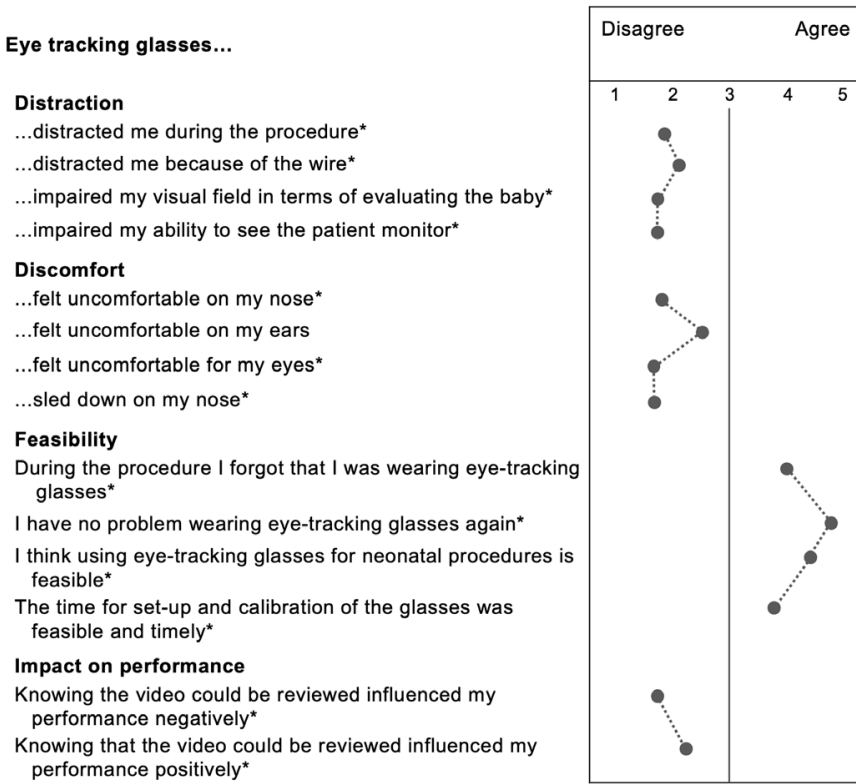

Figure 2 Proceduralists' subjective experience with the eye-tracking glasses during procedures. ${ }^{*} \mathrm{P}<0.05$ (Wilcoxon signed-rank test).

educational tool during COVID-19. In addition, there were no significant differences in responses between on-site and online observers. Qualitative feedback on what observers had learnt from the recordings included logistical issues, equipment and medication and awareness (see table 1).

\section{DISCUSSION}

Until now, video reflections on neonatal stabilisation at the Leiden Medical University Center were carried out with cameras installed in the room. ${ }^{7}$ The eye-tracking methodology was introduced during the COVID-19 pandemic for healthcare staff to witness procedures remotely while still having a first-person view. Our findings suggest the feasibility of wearing eye-tracking glasses during neonatal procedures and using recordings for subsequent video reflections with the healthcare staff. Former studies regarding the feasibility of wearing eye-tracking glasses in real neonatal resuscitation scenarios ${ }^{17}$ and simulated neonatal airway management scenarios ${ }^{14}$ yielded similar results. Most participants reported that wearing eye-tracking glasses was not disturbing or uncomfortable, in line with previous findings in simulation-based trainings. ${ }^{14}$ In another study concerning eyetracking in a paediatric trauma simulation, participants declared tolerating the glasses after a few minutes without being subjectively affected. $^{13}$

The use of point-of-view recordings..

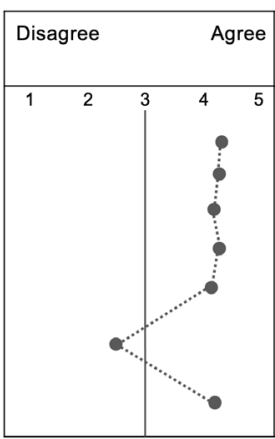

is feasible for our NICU

..was an educational benefit for $\mathrm{me}^{*}$

...helps with (virtual bedside) education during COVID-19 ..was done within a safe learning environment ${ }^{*}$

The first-person perspective was an addition to the educational experience

I was distracted by the first-person perspective during the audit $^{\star}$

Using the first-person perspective would be beneficial for reviewing other procedures too*

Figure 3 Observers' subjective experience with point-of-view recordings during audits. ${ }^{*} \mathrm{P}<0.05$ (one-sample t-test). NICU, neonatal intensive care unit. 
Table 1 Qualitative feedback on what observers had learnt after reviewing the recordings

\begin{tabular}{|c|c|}
\hline Category & Feedback \\
\hline Experience & Observers mentioned 'a lot of different variations in technique for placing lines'. \\
\hline Equipment, medication and timing & $\begin{array}{l}\text { To think about 'sedation balance' and 'comfort of the baby', meaning the sequence of sedation, removal of CPAP, mask/t-piece } \\
\text { application, ventilation and the 'use of different catheters (for MIST)' as well as the necessity to 'take more time to give MIST'. }\end{array}$ \\
\hline Environment and awareness & $\begin{array}{l}\text { To change 'the position of the baby or the monitor for a better visual' and think about 'the position of material in the room'. To } \\
\text { acknowledge to 'think about the blade you use'. }\end{array}$ \\
\hline Sterility & $\begin{array}{l}\text { Observers noticed 'violations in the sterile technique'. In terms of central line insertion, one observer mentioned that it is important to } \\
\text { 'check sterility, also for those around' and to think about 'how to improve sterility and increase awareness about sterility'. }\end{array}$ \\
\hline Point-of-view recording & $\begin{array}{l}\text { Point-of-view recording was especially 'worthwhile for learning umbilical catheterization and the crucial steps of the sterile technique'. } \\
\text { It helped to see the 'procedure through the eyes of the neonatologist' and 'how others operate'. }\end{array}$ \\
\hline Technique & $\begin{array}{l}\text { Some observers claimed that the videolaryngoscope blade } 1 \text { 'can be a problem because the light of the blade reflects on the tongue'. } \\
\text { It was further mentioned that the videolaryngoscope blade should be placed on the right side of the patient to avoid 'left-handed } \\
\text { insertion of the laryngoscope'. }\end{array}$ \\
\hline Education and training & $\begin{array}{l}\text { Eye-tracking technology is 'good to learn' and 'very nice to refresh' educational aspects. To acknowledge the 'differences in performing } \\
\text { the procedure' brings attention to changes such as 'start the procedure with the left hand and not with the right hand, otherwise you } \\
\text { have to change an extra time'. }\end{array}$ \\
\hline Technical issues & In some recordings it was 'difficult to see the head of the patient'. \\
\hline
\end{tabular}

CPAP, continuous positive airway pressure; MIST, minimally invasive surfactant therapy.

Furthermore, most observers stated that point-of-view video recordings could facilitate (virtual bedside) education during COVID-19 and perceived discussing the recordings from a firstperson perspective as highly beneficial. We also found that both on-site and online observers rated the eye-tracking recordings as an additional educational experience, particularly in medical training. Eye-tracking technology has been a tool for laparoscopic surgery and the learning process of square knot tying. The firstperson perspective gives trainees the possibility to identify critical areas, memorise the visual attention and adopt the experts' gaze patterns. ${ }^{18}$ A study by Brunye et al determined the eye fixation behaviour of experts and novices. ${ }^{10}$ Analysing the experts' procedures helped novices and students learn better strategies for successful interventions. Moreover, experts underlined the importance and need for innovative technology in order to offer trainees valuable education ${ }^{19}$ in times of COVID-19.

While we only recorded videos for a later review, it would have been possible to stream procedures for other professionals to assist from another room or their homes, as this option was proven to be practicable and inexpensive. ${ }^{20-22}$ We found no significant difference between online and on-site video-based reflections as both alternatives were judged 'equally good'. However, the online group consisted of only eight participants. Increasing this group could be an interesting next step to improve virtual bedside training. During the procedure, the supervisor could guide the trainee while maintaining distance thanks to the first-person view. Observers mentioned many key learning points, such as the overall logistics. In the first videobased reflection, as the proceduralist had to look over his/her shoulder to see the monitor, the staff discussed changing the proceduralist positioning to see the baby and the monitor from the same perspective. Adopting the new positioning in the six following recordings turned out to be more comfortable for the proceduralist and had an immediate effect on the video-based discussion. Furthermore, this technology could have a substantial educational benefit for nursing procedures, especially in single-room NICUs, where it might be more challenging to learn from other colleagues than open bay wards.

\section{Limitations}

As this was a feasibility study and reviewing neonatal stabilisation videos was already common among the local healthcare staff, it is difficult to generalise the results. The local NICU team was familiar with regular video-based reflections and was very supportive in creating a safe learning environment, even when conducting remote video-based reflections. However, this may be more challenging in NICUs where it is not common to hold team video-based reflections. Additional (multicentre) studies are needed to show the transfer to other NICUs. Observers also mentioned that the head of the patient was not always visible during intubations. Therefore, we reported the issue to the manufacturer, and the problem was solved within a month. While we did not include audio in our recordings, many observers recommended it for future video reflections. It was also mentioned that recording the vital signs monitor would also be helpful. At the moment, it is not possible to record the first-person view, the videolaryngoscope monitor, as well as the vital monitor with one device. However, the glasses might serve as an alternative view of what the participant is seeing in situations where another technology such as a videolaryngoscope is not available (ie, mouth and pharynx of the patient). A limitation for this at the moment is the fact that the video recording unit is over the eye level, which might make it difficult to see every detail of the pharynx. Therefore, new technology is necessary to improve this aspect and to review point-of-view neonatal procedures. Furthermore, eye-tracking glasses are still very expensive with one-time costs of about $€ 10.000$, depending on the company researchers choose.

\section{CONCLUSIONS}

We were able to show that it is feasible to use eye-tracking glasses for point-of-view recordings of neonatal procedures and use videos for reflections and educational sessions. Overall, observers stated that reviewing first-person videos has educational benefits, especially during the COVID-19 pandemic with social distancing measures reducing bedside teaching and logistics improvement opportunities.

Twitter Arjan B te Pas @None

Acknowledgements We acknowledge the support of healthcare providers at the Leiden Medical University Center in conducting this study. Viewpointsystem provided a non-restricted financial support for open-access charge.

Contributors MW, MCB and ABP conceptualised and designed the study. MW drafted the initial manuscript, collected and analysed the data and finalised the manuscript. MCB, SJ, RV, RSGMW, VB, EL and ABP collected the data and reviewed and revised the manuscript. PG helped with analysing the data. PG and $A B$ critically 
reviewed and revised the manuscript for important intellectual content. All authors approved the final manuscript as submitted and agreed to be accountable for all aspects of the work.

Funding The authors have not declared a specific grant for this research from any funding agency in the public, commercial or not-for-profit sectors.

Competing interests None declared.

Patient consent for publication Parental/guardian consent obtained.

Ethics approval The local IRB 'Medisch-Ethische Toetsingscommissie' committee in Leiden granted the exempt status for this study (ID: N20.135).

Provenance and peer review Not commissioned; externally peer reviewed.

Data availability statement All data relevant to the study are included in the article or uploaded as supplementary information.

Open access This is an open access article distributed in accordance with the Creative Commons Attribution Non Commercial (CC BY-NC 4.0) license, which permits others to distribute, remix, adapt, build upon this work non-commercially, and license their derivative works on different terms, provided the original work is properly cited, appropriate credit is given, any changes made indicated, and the use is non-commercial. See: http://creativecommons.org/licenses/by-nc/4.0/.

ORCID iDs

Michael Wagner http://orcid.org/0000-0003-0982-2310

Maria C den Boer http://orcid.org/0000-0003-4945-7303

Enrico Lopriore http://orcid.org/0000-0002-3513-5066

\section{REFERENCES}

1 Zhu N, Zhang D, Wang W, et al. A novel coronavirus from patients with pneumonia in China, 2019. N Engl J Med 2020;382:727-33.

2 Sen-Crowe B, McKenney M, Elkbuli A. Social distancing during the COVID-19 pandemic: staying home save lives. Am J Emerg Med 2020;38:1519-20.

3 Ferrel MN, Ryan JJ. The impact of COVID-19 on medical education. Cureus 2020;12:e7492

4 Wagner M, Jaki C, Löllgen RM, et al. Readiness for and response to coronavirus disease 2019 among pediatric healthcare providers: the role of simulation for pandemics and other disasters. Pediatr Crit Care Med 2021:22:e333-8.

5 Hofmann H, Harding C, Youm J, et al. Virtual bedside teaching rounds with patients with COVID-19. Med Educ 2020:54:959-60.
6 McKechnie T, Levin M, Zhou K, et al. Virtual surgical training during COVID-19: operating room simulation platforms accessible from home. Ann Surg 2020;272:e153-4.

7 den Boer MC, Houtlosser M, Foglia EE, et al. Benefits of recording and reviewing neonatal resuscitation: the providers' perspective. Arch Dis Child Fetal Neonatal Ed 2019:104:F528-34

8 den Boer MC, Martherus T, Houtlosser M, et al. Improving the quality of provided care: lessons learned from auditing neonatal stabilization. Front Pediatr 2020;8:560

9 Szulewski A, Howes D. Combining first-person video and gaze-tracking in medical simulation: a technical feasibility study. ScientificWorldJournal 2014;2014:1-4.

10 Brunyé TT, Drew T, Weaver DL, et al. A review of eye tracking for understanding and improving diagnostic interpretation. Cogn Res Princ Implic 2019;4:7.

$11 \mathrm{Kim}$ E. Potential of eye tracking technology for assessment of performance and medical education in the field of anesthesia. Korean J Anesthesiol 2018;71:253-4.

12 McNaughten B, Hart C, Gallagher S, et al. Clinicians' gaze behaviour in simulated paediatric emergencies. Arch Dis Child 2018;103:1146-9.

13 Damji $\mathrm{O}$, Lee-Nobbee $\mathrm{P}$, Borkenhagen $\mathrm{D}$, et al. Analysis of eye-tracking behaviours in a pediatric trauma simulation. CJEM 2019;21:138-40.

14 Wagner M, Gröpel P, Bibl K, et al. Eye-tracking during simulation-based neonatal airway management. Pediatr Res 2020;87:518-22.

15 Schilleman K, Witlox RS, van Vonderen JJ, et al. Auditing documentation on delivery room management using video and physiological recordings. Arch Dis Child Fetal Neonatal Ed 2014;99:F485-90.

16 Cuschieri S. The STROBE guidelines. Saudi J Anaesth 2019;13:31-4.

17 Law BHY, Cheung P-Y, Wagner M, et al. Analysis of neonatal resuscitation using eye tracking: a pilot study. Arch Dis Child Fetal Neonatal Ed 2018;103:F82-4.

18 Ashraf $\mathrm{H}$, Sodergren $\mathrm{MH}$, Merali $\mathrm{N}$, et al. Eye-tracking technology in medical education: a systematic review. Med Teach 2018:40:62-9.

19 Bloom DA, Reid JR, Cassady CI. Education in the time of COVID-19. Pediatr Radiol 2020:50:1055-8.

20 Jack MM, Gattozzi DA, Camarata PJ, et al. Live-Streaming Surgery for Medical Student Education - Educational Solutions in Neurosurgery During the COVID-19 Pandemic. J Surg Educ 2021;78:99-103.

21 Hiranaka T, Nakanishi Y, Fujishiro T, et al. The use of smart glasses for surgical video streaming. Surg Innov 2017;24:151-4.

22 Chaves RO, de Oliveira PAV, Rocha LC, et al. An innovative streaming video system with a point-of-view head camera transmission of surgeries to Smartphones and tablets: an educational utility. Surg Innov 2017;24:462-70. 